\title{
Expression of transient receptor potential vanilloid (TRPV) families 1, 2, 3 and 4 in the mouse olfactory epithelium*
}

\author{
Mohamed Khalifa Ahmed, Masaya Takumida, Takuya Ishibashi, \\ Takao Hamamoto, Katsuhiro Hirakawa
}

Department of Otorhinolaryngology, Head \& Neck Surgery, Division of Clinical Medical Science, Programs for Applied Biomedicine, Graduate School of Biomedical Sciences, Hiroshima University, Hiroshima city, Japan

\begin{abstract}
SUMMARY We investigated the expression of transient receptor potential vanilloid (TRPV)-1-4 in the mouse olfactory epithelium (OE) in comparison to its expression in respiratory epithelium. $C B A / J$ mice were used. The localization of TRPV-1, -2, -3 and -4 in the nasal mucosa was investigated using immunohistochemsitry and a double staining study for TRPV-1 and- 2 and SP was also carried out. TRPV-1-4, were expressed variably in the OE with a diffuse pattern in lamina propria, and were expressed in respiratory epithelium with strong positive expression in glandular cells of lamina propria. The double-staining study revealed coexpression of TRPVland -2 and substance $P(S P)$ in the trigeminal nerve fibers of the OE. Coexpression of TRPV-1 and SP was marked around the blood vessels and seromucinous gland of respiratory epithelium while TRPV-2 showed no co-localization. TRPV-1-4 were found to be localized in the mouse OE and respiratory epithelium. Our results suggest that TRPVS may play multiple roles in the $O E$, contributing to olfactory adaptation, olfactory/trigeminal interactions in nasal chemoreception and $O E$ homeostasis; they may also be involved in olfactory transduction as well as olfactory dysfunction secondary to sinonasal inflammatory disease. TRPVS in respiratory mucosa may play a significant role in nasal nociception, ciliary movement and the regulation of mucous secretion.
\end{abstract}

Key words: immunohistochemistry, mouse, nasal mucosa, olfactory, TRPV

\section{INTRODUCTION}

Transient receptor potential (TRP) ion channel family is the latest major ion channel family to be discovered. In the past few years, TRPV (vanilloid) subfamily has received increasing attention because of the significant role that some members of this group appear to play in sensory and non -sensory transduction. Mammalian TRPVs are divided into four groups based on structure and function: TRPV-1 / TRPV-2, TRPV-3, TRPV-4 and TRPV-5/TRPV-6. TRPV-1-4 are modestly permeable for $\mathrm{Ca}^{2+}$ while TRPV-5 and -6 are the only highly $\mathrm{Ca}^{2+}$ selective channels in the TRPV family. The TRPV subfamily is widely distributed in sensory and non-sensory cells. These channels can be activated by a diverse range of stimuli (osmotic/ mechanical stress, noxious chemicals and heat, endogenous mediators, etc.). The basic properties and functions of TRPVs, as well as emerging evidence, strongly suggest that some members of this subfamily are important for perception of pain, temperature sensitivity, osmotic regulation and maintenance of $\mathrm{Ca}^{2+}$ homeostasis ${ }^{(1-3)}$.
Sensory systems continuously adjust their sensitivity and kinetics based on history; they are responsible for sensory homeostasis and certain types of control, which are essential for all chemosensory behaviours ${ }^{(4)}$. They are typically coupled with the activation of ion channels, thereby transducing specific stimuli into electrical signals. These sensory systems are highly specialized for sensing changes in the external environment; all cells in both lower and higher organisms have the innate ability to sense and respond to alterations in various chemical and physical stimuli in the local microenvironment of the cells. Such sensing and responding to microenvironmental changes are critical for the regulation of the feedback control systems at the cellular level that, in turn, underlie higher- order control systems and maintenance of body homeostasis ${ }^{(5)}$. Many studies have revealed abundant and wide expression of TRPV-1-4 in different cell types and have suggested their specific roles in numerous fundamental cell functions in these delicate sensory systems ${ }^{(1-3)}$. Since the identification of the vallinoid channels, an ongoing objective has been to identify its expression and 
possible significance in different tissues. The olfactory system is unique in that olfactory sensory neurons are embedded in the epithelium in the nasal cavity and directly face the external environment and in which. To date, little is known regarding the expression and potential roles of TRPV-1-4 in the olfactory epithelium (OE).

The aim of the present study was to reveal the expression of TRPV-1-4 in mouse OE in comparison to its expression in the respiratory epithelium in order to establish its possible physiological significance in the olfactory system.

\section{MATERIALS AND METHODS}

\section{Animals and tissue removal}

We used a healthy, normal, 8-week-old CBA/J mouse with a body weight of $25 \mathrm{~g}$ and normal Preyer's reflex. The care and use of the mouse was approved by the Animal Experimentation Committee of the Hiroshima University School of Medicine (permit no. A07-96) and was carried out in accordance with the guide to Animal Experimentation of Hiroshima University and the guidelines established by the Committee on Research facilities for Laboratory Animal Science of the Hiroshima University School of Medicine. Mice were deeply anesthetized with diethyl ether and decapitated. The nose with palate was then dissected and immersed in $4 \%$ paraformaldehyde in $0.1 \mathrm{M}$ phosphate buffer solution (PBS), pH 7.4 for 2 hours. The nasal mucosa (respiratory and olfacto-
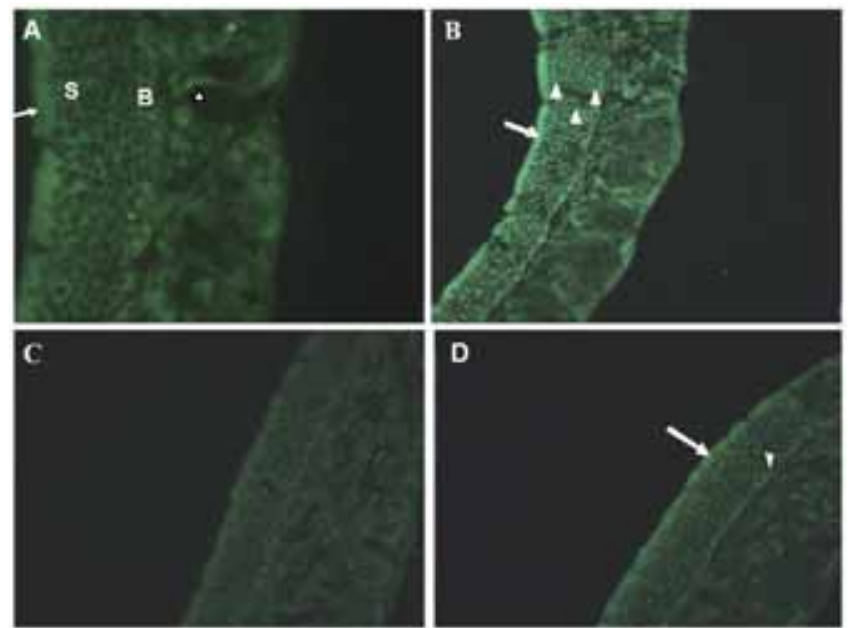

Figure 1. The mouse olfactory epithelium (A) TRPV-1 labelling is noted in the olfactory cilia (arrow), epithelial layer [S, supporting cell layer; O, olfactory receptor neurons; $\mathrm{B}$, basal cell layer] and trigeminal nerve fibers in lamina propria (arrowhead). (B) TRPV-2 labelling is observed in the cilia, the epithelial cell layer and the lamina propria with marked fluorescence in theolfactory cilia (arrow) and plasma membrane in the zone of basal cells, olfactory receptor neurons and supporting cells (3 arrowheads). (C) Immunofluorescence to TRPV-3 is homogenous in theolfactory cilia, epithelial cell layer and lamina propria. (D) Immunoreactivity to TRPV-4 is observed in the olfactory cilia, epithelial cell layer and lamina propria with obvious fluorescence in theolfactory cilia (arrow) and basal cell layer (arrowhead). ry) were dissected and cryoprotected in 10, 15 and 20\% sucrose in $0.1 \mathrm{M}$ PBS for 24 hours.

\section{Immunohistochemistry}

The specimens were frozen in optimal cutting temperature (OCT) mounting medium (Sakura Fine Technical Co., Ltd., Tokyo, Japan), serially sectioned on a cryostat at $4 \mu \mathrm{m}$, and mounted on glass slides. After pretreatment with blocking serum, the specimens were incubated with a rabbit polyclonal antibody to TRPV-1 (Transgenic, Inc., Kumamoto, Japan) at a dilution of $0.1 \mu \mathrm{g} / \mathrm{ml}$, a rabbit polyclonal antibody to TRPV-2 (Abcam, Tokyo, Japan) diluted 1:1000, a goat polyclonal antibody to TRPV-3 (Santa Cruz Biotechnology Inc., Santa Cruz, CA, USA), diluted 1:50 or with a rabbit polyclonal antibody to TRPV-4 (Alomone Labs, Ltd., Jerusalem, Israel), diluted 1:200 in $0.3 \%$ Triton X-100 containing PBS at $4{ }^{\circ} \mathrm{C}$ for $48 \mathrm{~h}$. The specimens were then washed in PBS and incubated for 1 hour with Alexa-Fluor488 goat anti-rabbit or donkey anti-goat secondary antibodies (1:500) (Molecular Probes, Eugene, OR, USA). The sections were washed and cover-slipped with DakoCytomation Fluorescent Mounting Medium (DakoCytomatia, Carpinteria, CA, USA).

For the double staining studies, the sections were first stained with mouse monoclonal antibody to substance P (SP; Immundiagnostik AG, Benshwim, Germany) at a dilution of 1:300 followed by secondary antibodies conjugated with
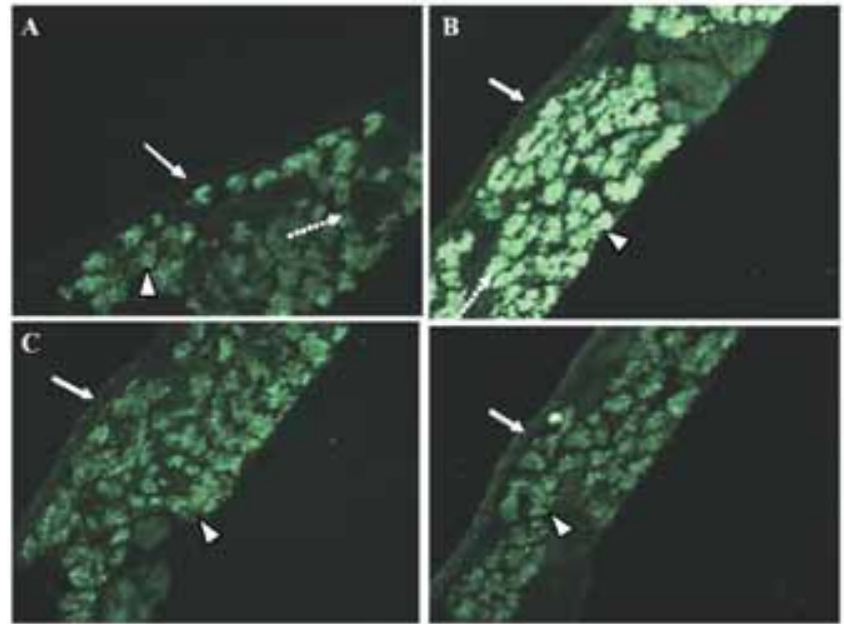

Figure 2. The respiratory mouse epithelium. (A) TRPV-1 labelling is noted in the lamina propria; the seromucinous gland (arrowhead) and capillary endothelial cell (dashed arrow) show strong positive expression while the epithelial layer shows no significant fluorescence (arrow) (B) immunoreactivity to TRPV-2 is strongly positive in the seromucinous gland of the lamina propria (arrowhead) and capillary endothelial cells (dashed arrow) with weak fluorescence in the epithelium (arrow). (C) The distribution of TRPV-3 is almost identical to that of TRPV-2, with the seromucinous gland showing strong positive expression (arrowhead), while the epithelium shows weak expression (arrow). (D) TRPV-4 shows significant fluorescence in the seromucinous gland (arrowhead) in comparison to the epithelium (arrow). 
Alexa568 goat anti-mouse and then stained for TRPV-1, TRPV-2 with secondary antibodies conjugated with Alexa488. The specimens were viewed under a Nikon fluorescence microscope (Eclipse E600) equipped with an appropriate filter set. Fluorescence analogue images were obtained via an intensified digital colour charge-coupled device camera (C4742-95; Hamamatsu Photonics) and stored as digital images using IP Lab Spectrum software (version 3;0 Signal Analytics Corporation, Vienna, VA, USA).

\section{RESULTS}

We tested TRPV-1-4 in the olfactory and respiratory regions of mouse nasal tissues by immunofluoresence staining of the frozen section. TRPV-1-4 were found to be expressed variably in the OE with a diffuse pattern in lamina propria as shown in (Figure 1). Additionally, TRPV-1-4 were expressed in the respiratory epithelium with strongly positive expression in the glandular cells of the lamina propria (Figure 2).

\section{TRPV-1}

In the OE, TRPV-1 immunofluorescence was evident in the olfactory cilia with variable labelling in the basal cells, supporting cells and olfactory receptor neurons (ORNs). Diffuse labelling was observed in the lamina propria. In contrast, TRPV-1 in the respiratory epithelium showed strongly positive expression in the seromucinous glands and capillary endothelial cells of the lamina propria with weakly positive to negative expression in the epithelial layer (Figure 2A).
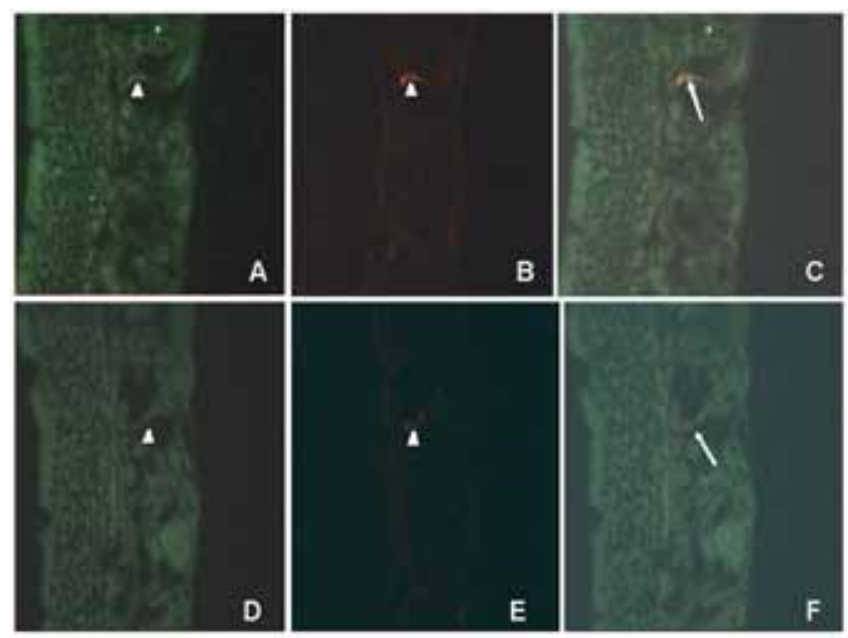

Figure 3. Double labelling for SP (red) and TRPV-1 and -2 (green) in olfactory mucosa. (A) TRPV1 immunoreactive nerve fibers in the lamina propria (arrowhead). (B) SP nerve fibers (arrowhead). (C) Colocalization of TRPV-1 with SP revealed as an orange colour in the trigeminal nerve fibers (arrow). (D) TRPV-2 immunoreactive nerve fibers (arrowhead). (E) SP nerve fibers in the lamina propria (arrowhead). (F) Coexpression of TRPV-2 and SP show as an orange colour (arrow).

\section{$T R P V-2$}

TRPV-2 showed marked positive expression in the olfactory cilia with variable and diffuse expression in the rest of the epithelial layer (basal cells, ORNs and supporting cells). TRPV2 was also evident in lamina propria (Figure 1B). In comparison, TRPV-2 showed strongly positive expression in lamina propria of the respiratory epithelium, particularly in the glandular cells and capillary endothelial cells. Immunoreactivity to TRPV-2 was also observed in the epithelial cells (Figure 2B).

\section{$T R P V-3$}

TRPV-3 showed almost equal expression across OE (olfactory cilia, basal cells, ORNs, supporting cells and lamina propria) with comparatively weak labelling compared to that of TRPV1and TRPV-2 (Figure 1C). In the respiratory epithelium, immunoreactivity to TRPV-3 was observed in both the epithelial layer and the lamina propria with marked expression in the lamina propria (seromucinous glands and capillary endothelial cells) (Figure 2C).

\section{$T R P V-4$}

Immnoreactivity to TRPV-4 was observed in the epithelial layer of the OE with marked expression in the olfactory cilia and basal cell layer. TRPV-4 labelling was comparatively weak in the lamina propria (Figure 1D). In the respiratory epithelium TRPV-4 immnoreactivity was strongly positive in the lamina propria compared to that in the epithelial layer (Figure 2D).
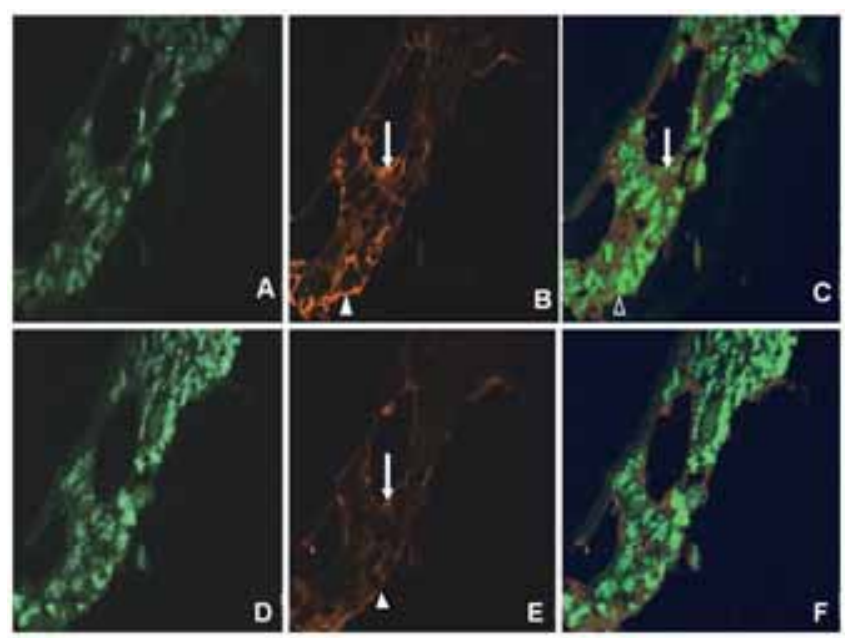

Figure 4. Double labelling for SP (red) and TRPV-1 and -2 (green) in the respiratory mucosa. (A) TRPV-1 immunoflourescence (green). (B) SP immunorective nerve fibers scattered around capillary blood vessels (arrowhead) and seromucinous gland (arrow). (C) Orange fluorescence denoting coexpression TRPV1 (green) and SP (red) around blood vessels (arrowhead) and seromucinous gland (arrow). (D) TRPV-2 immunoflourescence (green). (E) SP immunoreactive nerve fibers (red) in the lamina propria around the capillary vessels (arrowhead) and seromucinous glands (arrow). (F) Double labelling for TRPV2 (green) and SP (red) revealed no coexpression. 


\section{Double staining study}

The double staining study revealed coexpression of TRPV1and TRPV-2 with SP in trigeminal nerve fibres of the OE (Figure 3). On the other hand, coexpression of TRPV-1 and SP was observed around the blood vessels and seromucinous gland of the respiratory epithelium, while TRPV-2 showed no colocalization with SP (Figure 4).

\section{DISCUSSION}

The present study revealed TRPV-1 immunoreactivities in olfactory cilia. TRPV-1 channels were recently found to directly bind $\mathrm{CaM}$ (calmodulin) at two sites in both a $\mathrm{Ca}^{2+}$-dependent and $\mathrm{Ca}^{2+}$-independent manner. $\mathrm{Ca}^{2+} / \mathrm{CaM}$ (calcium /calmodulin) is involved in the desensitization of TRPV-1 channels. This desensitization is triggered by the activation of TRPV-1, which leads to an elevation in intracellular free $\mathrm{Ca}^{2+}$ levels ${ }^{\left({ }^{6}\right)}$. Much interest has been focused on the precise mechanisms by which $\mathrm{Ca}^{2+}$ in olfactory system can downregulate cyclic nucleotide-gated (CNG) channels activity. However, definitive evidence on the nature of endogenous molecules mediating $\mathrm{Ca}^{2+}$-dependent $\mathrm{CNG}$ channels modulation and short-term adaptation (STA) is still lacking ${ }^{(7)}$. In view of these findings, we hypothesize that TRPV-1 may have a role in CNG channels modulation and STA of olfactory system. The rapid termination of stimulus-induced receptor potential is thought to enable ORNs to recover sufficiently to fire action potentials to subsequent stimulation ${ }^{(8)}$.

The present study revealed coexpression of TRPV-1 and SP in trigeminal nerve fibres in the lamina propria of $\mathrm{OE}$. Electrophysiological studies have indicated that spontaneous activity of the olfactory receptor cells can be modified via a local axon reflex triggered by odours and inducing the release of SP with a concomitant analgesic effect, or via other peptides from trigeminal fibres innervating the $\mathrm{OE}^{(9,10)}$. There is also evidence also that TRPV-1 and SP fibres are expressed in the olfactory bulb ${ }^{(11,12)}$. Another study raises the possibility that the trigeminal and olfactory systems could also interact at the central level ${ }^{(10)}$. We speculate that TRPV-1may play a role in peripheral and central olfactory/trigeminal interaction in nasal chemoreception through the peripheral and central release of SP.

The present study showed significant TRPV2 immunofluorescence in basal cells, ORNs and supporting cells. As TRPV-2 is expressed in neuronal tissue as well as stretch-sensitive cells, it may be involved in stretch-activated $\mathrm{Ca}^{2+}$ influx in these cells $(3,13,14)$. Thus a role for TRPV-2 as an osmotic and stretch sensor in basal cells, ORNs and supporting cells is anticipated. Furthermore, TRPV-2 showed co expression with SP similar to that of TRPV-1. We therefore suggest that TRPV-2 may play a role in the peripheral olfactory/trigeminal interactions in nasal chemoreception ${ }^{(10)}$.
The present study showed significant fluorescence for TRPV-3 in olfactory cilia, basal cells, ORNs and lamina propria of OE. TRPV-3 is unique in that its activity is sensitized upon repetitive stimulations. As new evidence for the involvement of TRP channels in chemesthesis is mounting, it is highly likely that the sensitization property of the TRPV-3 channel may underlie the mechanism of enhanced oral and nasal sensitization to successive exposures of certain odours, flavour, and irritants ${ }^{(2,15)}$. Based on these findings we suggest that TRPV-3 act as chemoesthetic receptor and that therefore, it may play a role in allergic rhinitis caused by successive exposure to certain odours.

The present study showed positive immunofluorescence for TRPV-4 in olfactory cilia, basal cells, ORNs, supporting cells and lamina propria in the OE. It is now recognized that TRPV4 controls the secretion of antidiuretic hormones and in turn, controls water balance ${ }^{(2,5)}$. In addition, an extensive network of aquaporin (AQP) expression is found in the olfactory mucosa. AQP5 may play a role in the secretion of the seromucinous glands to maintain the surface microenvironment around dendrites of the olfactory sensory cells with odorants' receptors ${ }^{(16)}$. A previous study found that TRPV-4 participates in the hypotonic reduction of AQP5 ${ }^{(17)}$. These findings suggest that TRPV-4 has a direct relevance to homeostasis in the OE.

\section{TRPV-1-4 as complex sensory integrators of diverse stimuli} The present study revealed wide expression of TRPV1-4 in the olfactory cilia. It is well recognized that $\mathrm{Ca}^{2+}$ is an important signal transduction molecule in all cells, and play a critical role in olfactory transduction ${ }^{(4,18)}$. The present study revealed wide expression of TRPV1-4 in the olfactory cilia. Hence, because of the high permeability to $\mathrm{Ca}^{2+}$ and their sensitivities to various physiological stimuli, a role for these channels in olfactory transduction seems highly likely. However, some questions remain unclear and need to be elucidated. Are TRPVs activated by all odours? Do osmolarity and pH of odiferous substance play a role in their activation?

\section{Inflammation, TRPV-1-4 and the olfactory process}

With respect to inflammation, a viral upper respiratory infection (URI) is one of the most commonly identified causes of persistent olfactory dysfunction ${ }^{(19)}$. Additionally, chronic sinusitis is a major cause of smell loss ${ }^{(20)}$. The successful treatment of sinusitis and URI may resolve the smell loss, but the success of medical and surgical treatment in resolving loss of smell is variable. The failure to resolve the problem can be frustrating for both the patient and the physician. Anosmia secondary to sinusitis is assumed to result from the inability of odorant molecules to access the olfactory mucosa, which is believed to remain histologically intact despite the aggressive inflammatory process in the respiratory regions of the nose. Clinical studies have failed to provide support for this hypothesis however, as there is little correlation between nasal resis- 
tance and the degree of olfactory dysfunction. Kern showed, in his histopathologic study of neuroepithelium in chronic sinusitis patients, that anosmia secondary to sinonasal inflammation is both a transport and sensory disorder ${ }^{(21)}$. Leopold also has pointed out that an inflammatory product released while fighting the URI, or an autoimmune process, could be producing epithelial changes and causing an olfactory loss ${ }^{(22)}$. Therefore, several questions remain considering the true pathophysiology of smell loss following URI and chronic sinusitis. In the case of inflammation, a combination of endogenous vanilloids and inflammatory mediators may act on TRPVs, in turn initiating inflammatory cascade. Inflammatory mediators released by lymphocytes and macrophages, trigger hypersecretion in respiratory and seromucinous glands. Olfactory mucous produced by seromucinous glands, is a highly specialized substance that is vastly different from nasal respiratory mucous, which serves a function analogous to that of cochlear endolymph. Hypersecretion would likely alter the ion concentration of olfactory mucous, affecting the microenvironment of olfactory neurons and the transduction process. In addition, there is evidence that $\mathrm{Ca}^{2+}$ in mucous may modulate sensitivity to cAMP in vivo; therefore, decreasing nasal mucous $\mathrm{Ca}^{2+}$ improves hyposmia ${ }^{(21,23)}$. The present study showed wide expression of TRPV1-4 in the OE, suggesting that TRPV channels function as novel molecular integrators of diverse microenvironmental stimuli. The present findings are useful in improving our understanding of the pathophysiology of olfactory dysfunction secondary to sinonasal inflammatory diseases.

\section{TRPV1-4 in respiratory mucosa}

The present study found positive expression of TRPV-1 and TRPV-2 in the epithelium with strong positive expression in the lamina propria, and a double staining study revealed significant immunofluorescence for TRPV-1 and TRPV-2 in seromucinous gland of the respiratory epithelium while SP immunofluorescences were observed around the seromucinous gland and capillary blood vessels. It has been reported that TRPV-1 positive cells in human nasal mucosa were found in epithelial cells, vascular endothelial cells, and submucosal glands and cells. This wide distribution may indicate a regulatory role in the production of cytokines and in the apoptosis of endothelial cells. It may also influence the secretory function of the human upper airway ${ }^{(24)}$. TRPV-1 and SP showed diverse coexpression around the seromucinous gland and capillary blood vessels. These findings are consistent with previous results showing that SP-containing nerve fibres are found around the nasal arterial and venous vessels, gland acini and in the epithelium. There is growing evidence that SP participates in allergic reaction in the nasal mucosa, and that patients with allergic rhinitis have higher tissue concentration of SP than healthy individuals ${ }^{(25,26)}$. The present results suggest that TRPV-1 plays a role in nasal nociception. In addition, TRPV-1 and -2 may play a role in the regulation of mucous secretion.
The present study also showed strong positive expression for TRPV-3 particularly in the glandular cells. TRPV3 act as molecular target of some allergens in the nose and may relay chemosensory information from the nasal epithelium to trigeminal nerve ending. Thus we suggest that TRPV3 may play a modulatory role in mucous secretion in the respiratory mucosa $^{(15)}$.

The present results showed significant immunofluorescence of TRPV-4 in the respiratory cilia and epithelium with strong positive expression in the glandular cells in the lamina propria. TRPV-4 mRNA is expressed in the respiratory epithelium and secretory cells in sinus mucosal biopsy specimens obtained from patients with chronic rhinosinusitis, patients with chronic rhinosinusitis with nasal polyps and healthy controls ${ }^{(27)}$. TRPV-4 has also been reported to play a role in the regulation of epithelial barrier function, membrane permeability; and water homeostasis in the respiratory epithelia ${ }^{(28)}$.

It is expected that TRPV4 may play a role in ciliary movement, respiratory homeostasis, and that it may be implicated in the pathogenesis of chronic rhinosinusitis ${ }^{(27)}$.

Regarding the antibody specificity, the specificity in our study is determined by both a negative control (no labelling could be detected by eliminating the primary antibody) and a positive control (respiratory nasal mucosa).

In summary, we detected localization of TRPV-1-4 in the mouse $\mathrm{OE}$ and respiratory epithelium. We believe the present study to be the first to reveal the expression and possible physiological roles of TRPV-1-4 in the olfactory mucosa. However, it must be noted that these findings are still preliminary and that further in vivo and in vitro studies are needed to establish the physiological significance of the immunolocalization of TRPV-1-4 and its implications for pathophysiological entities in the OE.

\section{ACKNOWLEDGEMENTS}

The authors would like to thank Ms. Ai Kashima for her excellent technical assistance.

\section{REFERENCES}

1. Hardie RC. TRP channels and lipids: from Drosophila to mammalian physiology. J Physiol 2007; 578: 9-24.

2. Pedersen SF, Owsianik G, Nilius B. TRP channels: an overview. Cell Calcium 2005; 38: 233-252.

3. O'Neil RG, Brown RC. The Vanilloid receptor family of Calcium permeable channels: Molecular integrators of microenvironmental stimuli. News Physiol Sci 2003; 18: 226-231.

4. Bargmann CI. Chemosensation in C. elegans, WormBook, ed. The C. elegans Research Community 2006; 1-29.

5. O'Neil RG, Heller S. The mechanosensitive nature of TRPV channels. Pflugers Arch-Eur J Physiol 2005; 451: 193-203.

6. Vyklicky L, Novakova-Tousova K, Benedikt J, Samad A, Touska F, Vlachova V. Calcium-dependent desensitization of vanilloid receptor TRPV1: a mechanism possibly involved in analgesia induced by topical application of capsaicin. Physiol Res [Epub ahead of print] 2008; 57: 1-25. 
7. Zufall F, Leinders-Zufall T. The cellular and molecular basis of odor adaptation. Chem Senses 2000; 25: 473-381.

8. Song Y, Cygnar KD, Sagdulloev B. et al. Olfactory CNG channels desensitization by $\mathrm{Ca}^{2+} / \mathrm{CaM}$ via the $\mathrm{B} 1 \mathrm{~b}$ subunit affects response termination but not sensitivity to recurring stimulation. Neuron 2008; 58: 377-386.

9. Silver WL, Mason JR, Marshall DA, Maruniak JO. Rat trigeminal, olfactory and taste responses after capsaicin desensitization. Brain Research 1985; 333: 45-54.

10. Brand G. Olfactory/trigeminal interactions in nasal chemoreception. Neurosci Biobehavioral Rev 2006; 30: 908-917.

11. Toth A, Boczan J, Kedei N, et al. Expression and distribution of vanilloid receptor 1 (TRPV1) in the adult rat brain. Brain Res Mol Brain Res 2005; 135: 162-168.

12. Finger TE, Bottger B. Peripheral peptidergic fibers of the trigeminal nerve in the olfactory bulb of the rat. J Comp Neurol 1993; 344: 117-124.

13. Folgering JHA, Sharif-Noeini R, Dedman A, Patel A, Delmas P, Honore E. Molecular basis of the mammalian pressure-sensitive ion channels: Focus on vascular mechanotransduction. Progress Biophys Mol Biol 2008: 1-16.

14. Liedtke W. Role of TRPV ion channels in sensory transduction of osmotic stimuli in mammals. Exp Physiol 2006: 507-512.

15. Xiao R, Tang J, Wang C, Calton CK, Tian J, Zhu MX. Calcium plays a central role in the sensitization of TRPV3 channel to repetitive stimulations. J Biol Chem 2008; 283: 6162-6174.

16. Ablimit A, Matsuzaki T, Tajika Y, Aoki T, Hagiwara H, Takata K Immunolocalization of water channel aquaporins in the nasal olfactory mucosa. Arch Histol Cytol 2006; 69: 1-12.

17. Sidhaye VK, Guler AD, Schweitzer KS, D'Alessio F, Caterina MJ, King LS.Transient receptor potential vanilloid 4 regulates aquaporin-5 abundance under hypotonic conditions. PANS 2006; 103: 4747-4752.

18. Tobin D M, Madsen DM, Kahn-Kirby A, et al. Combinatorial expression of TRPV channel proteins defines their sensory functions and subcellular localization in C. elegans. Neuron 2002; 35: 307-318.

19. Seiden AM. Post viral olfactory loss. Otolaryngol Clin N Am 2004; 37: 1159-1166.

20. Mann NM, Lafreniere D. Anosmia and nasal sinus disease. Otolaryngol Clin N Am 2004; 37: 289-300.

21. Kern RC. Chronic sinusitis and anosmia: pathologic changes in the olfactory mucosa. Laryngoscope 2000; 110: 1071-1077.

22. Leopold D. Aperplexing olfactory loss. Arch. Otolaryngol 2000; 126: $80-83$
23. Panagiotopoulos G, Naxakis S, Papavasiliou A, Filipakis K, Papatheodorou G, Goumas P. Decreasing nasal mucus $\mathrm{Ca}^{2+}$ improves hyposmia. Rhinology 2005; 43: 130-134.

24. Seki N, Shirasaki H, Kikuchi M, Sakamoto T, Watanabe N Himi T. Expression and localization of TRPV1 in human nasal mucosa. Rhinology 2006; 44: 128-134.

25. Hanf G, Schierhon K, Brunnee T, Noga O, Verges D, Kunkel G. Substance $\mathrm{P}$ induced histamine release from nasal mucosa of subjects with and without allergic rhinitis. Inflamm Res 2000; 49: 520523.

26. Ding QT, Groneberg DA, Mingomataj E, et al. Expression of substance $\mathrm{P}$ and vanilloid receptor (VR1) in trigeminal sensory neurons projecting to the mouse nasal mucosa. Neuropeptides 2003; 37: 245-250.

27. Bhargave G, Woodworth BA, Xiong G, Wolfe SG, Antunes MB, Cohen NA. Transient receptor potential vanilloid type 4 channel expression in chronic rhinosinusitis. Am J Rhinol 2008; 22: 7-12.

28. Sidhaye VK, Schweitzer KS, Caterina MJ, Shimoda L, King SL. Shear stress regulates aquaporin-5 and airway epithelial barrier function. PANS 2008; 105: 3345-3350.

Katsuhiro Hirakawa, M.D., Ph.D.

Department of Otorhinolaryngology,

Head \& Neck Surgery

Graduate School of Biomedical Sciences,

Hiroshima University

1-2-3 Kasumi, Minami-ku

Hiroshima city, Hiroshima

734-8551 Japan

Tel: +81-82-2575252

Fax: +81-82-257525

E-mail address: katsuhw@hiroshima-u.ac.jp

Mohamed Khalifa Ahmed

khalifa369@yahoo.com

Home University: Assiut University, Egypt 